\title{
Could cognitive estimation ability be a measure of cognitive reserve?
}

\author{
Guido E. D'Aniello ${ }^{1 *}$, Gianluca Castelnuovo ${ }^{1,2}$ and Federica Scarpina ${ }^{1,3}$ \\ ${ }^{1}$ Psychology Research Laboratory, Istituto di Ricovero e Cura a Carattere Scientifico Istituto Auxologico Italiano, Ospedale \\ San Giuseppe, Piancavallo, Italy, ${ }^{2}$ Department of Psychology, Università Cattolica del Sacro Cuore, Milan, Italy, ${ }^{3}$ "Rita Levi \\ Montalcini" Department of Neuroscience, University of Turin, Turin, Italy
}

Keywords: cognitive estimation, cognitive reserve, executive functions, crystallized intelligence, neuropsychological assessment

\section{The Cognitive Estimation Ability: A Matter of Discuss}

OPEN ACCESS

Edited by:

Snehlata Jaswal,

Indian Institute of Technology Jodhpur,

India

Reviewed by:

Patrizia Silvia Bisiacchi

University of Padova, Italy

${ }^{*}$ Correspondence: D'Aniello Guido Edoardo, g.daniello@auxologico.it

Specialty section:

This article was submitted to

Cognitive Science, a section of the journal

Frontiers in Psychology

Received: 11 March 2015

Accepted: 23 April 2015

Published: 07 May 2015

Citation:

D'Aniello GE, Castelnuovo G and Scarpina $F$ (2015) Could cognitive estimation ability be a measure of

cognitive reserve?

Front. Psychol. 6:608.

doi: 10.3389/fpsyg.2015.00608
The first attempt to measure cognitive estimation abilities was made by Shallice and Evans (1978), who developed the Cognitive Estimation Test, consisting of a set of questions which requires appropriate reasoning abilities. The authors stressed the link between executive functions and estimation skills, finding out that patients with frontal lobe damages performed poorly on the proposed task; although the relation between cognitive estimation ability and executive functions is been a matter of discussion (Spreen and Strauss, 1998; Appollonio et al., 2003; Barabassy et al., 2010; D’Aniello et al., 2015), it has been recently supported by MacPherson et al. (2014), who developed a new version of the cognitive estimation test, proving its suitability for assessing executive dysfunction; in a nutshell, it seems that the process of estimation requires a complex pattern of abilities, including executive functions.

Brand et al. (2003) proposed that cognitive estimation would follow a route from a specific representation in the working memory to an activation of information from the long-term memory and- subsequently-a "plausibility check" of the generated answer by a central processing control. According to this, a cognitive estimation task would involve (i) a central processing control, which defines the appropriate strategies to solve the problem, (ii) working memory, which applies these strategies, (iii) long term declarative memory, where the necessary information for answering the task are stored.

We accounted this idea in a detailed cognitive model (Figure 1) that may enclose the cognitive domains involved in the estimation process; furthermore, a more detailed characterization of the central processing control described by Brand et al. (2003) has been provided.

\section{A Revised Model}

\section{Part I: Semantic Knowledge and its Relation with the Hypothesis of Cognitive Reserve}

A verbal question relative to a cognitive estimation (e.g., "what is the average length of a man's spine?") should firstly activate the central executive of working memory (Baddeley and Hitch, 1974; Baddeley, 2000), which is crucial in order to set a link between the information provided by the question and the semantic knowledge. The established interface between working memory and semantic knowledge is exploited by the first level of executive processing, the reasoning abilities: the result of this stage is the formulation of a hypothesis. Depending on the question's contents (e.g., time, quantity, size or other type of information) the reasoning system would recruit additional executive skills, such as spatial abilities, planning, problem solving, numerical skills, experiences, and mental imagery (Horacek et al., 2010). The hypothesis generated by this complex process, in which 


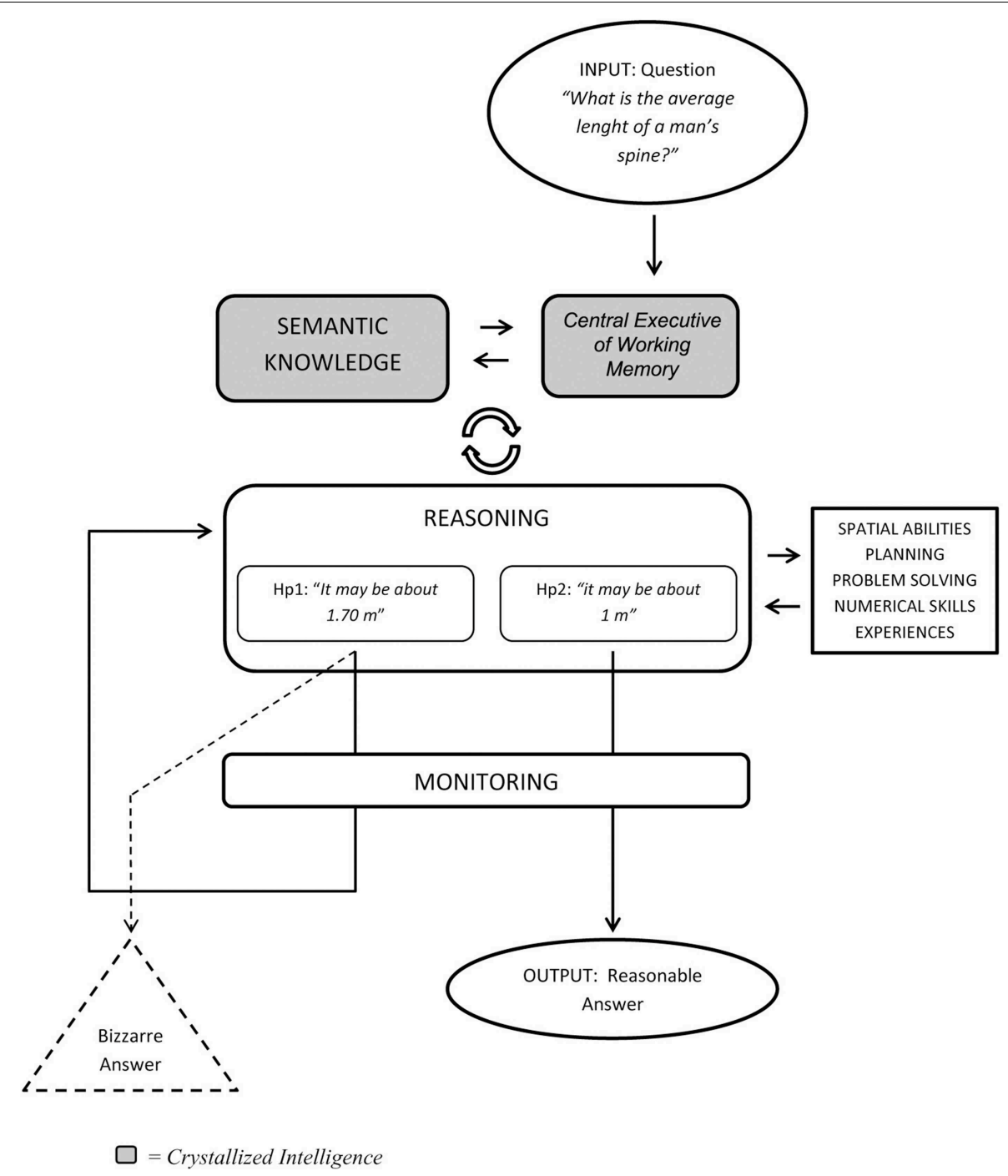

FIGURE 1 | Model of the cognitive estimation process.

the previous cognitive modules interact with each other, is then checked by the second main level of executive processing, that is, monitoring: at this stage, the formulated hypothesis is compared with all the information gleaned during the process in order to verify its consistency and-once accepted-proposed as the final answer. In case the hypothesis is considered as an inconsistent or inadequate one, it is sent back to the reasoning process. This process substantially differs from reasoning abilities applied on unknown information in novel contexts (fluid intelligence, Cattell, 1967), about which, for example, the Weigl Color-Form Sorting Test (Goldstein and Scheerer, 1941) would represent an example (Hobson et al., 2007). On the contrary, cognitive estimation is strictly linked (and depends) to a certain amount of previously acquired knowledge and experience as well as the ability to use it (Della Sala et al., 2003): this combination of abilities could be defined as crystallized intelligence (Cattell, 1967).
The definition of crystallized intelligence as stated above is surprisingly similar to the widely known concept of Cognitive Reserve. According to Stern (2009), cognitive reserve can be defined as the amount of cognitive loss that can be sustained by brain-damaged patients before reaching a threshold at which a clear cognitive impairment becomes evident.

The estimation of cognitive reserve has been traditionally conducted using tests of crystallized intelligence, such as vocabulary, reading ability and general knowledge (Cattell, 1971; Sumowski et al., 2010). The Wechsler Adult Intelligence ScaleInformation Subtest (Wechsler, 1997) has been widely used as an appropriate proxy for cognitive reserve, although it was originally intended as an instrument for assessing crystallized intelligence (Franchow et al., 2013): as confirmed by Stern (2007), measures of crystallized intelligence could provide a reliable instrument for assessing one's cognitive reserve, because they serve in capturing mature cognitive ability, even at a premorbid level (Sachdev 
and Valenzuela, 2009). What seems to be increasingly clear is that crystallized intelligence could represent almost part of the cognitive reserve construct, which is likely to comprise other factors including educational attainment (Stern et al., 1992; Stern, 2002; Tucker and Stern, 2011) and certain cognitive domains such as verbal fluency, reasoning, attention and working memory functions (Roldán-Tapia et al., 2012).

Given these findings, we hypothesize that assessing cognitive estimation abilities might be considered a reliable strategy for measuring important aspects of cognitive reserve: in fact, it could be used as a measure of the person's body of knowledge and experiences (Della Sala et al., 2004), independently from aging (Della Sala et al., 2004; MacPherson et al., 2014), and-at the same time-as a sensitive instrument for assessing certain cognitive domains such as working memory and semantic knowledge (D'Aniello et al., 2015). The hypothesis that semantic knowledge is part of the cognitive estimation ability would be confirmed by studies relative to patients with Alzheimer's Disease (Della Sala et al., 2004; Levinoff et al., 2006; Barabassy et al., 2007; Khodarahimi and Rasti, 2011), Mild Cognitive Impairment (Levinoff et al., 2006), and Korsakoff's syndrome (Taylor and O'Carroll, 1995; Brand et al., 2003), in which higher amount of errors in the estimation process comparing to healthy groups were reported.

\section{Part II: Reasoning Ability and the Relationship with Bizarre Answers}

The second part of the model regards reasoning, i.e., the ability to put together the information from other cognitive domain into the cognitive estimation, and monitoring, i.e., the process involves a systematic and continuous way of the reasoning process and to furnish a feedback about the results. According to our model, if the primary output from the reasoning process is not put through the monitoring stage and, for example, it is not checked with further everyday information (Shallice and Evans, 1978), but is directly provided as a result of the estimation, the final output would be a bizarre answer: it is an unreasonable and extremely

\section{References}

Appollonio, I. M., Russo, A., Isella, V., Forapani, E., Villa, M. L., Piolti, R., et al. (2003). Cognitive [correction of cognitve] estimation: comparison of two tests in nondemented parkinsonian patients. Neurol. Sci. 24, 153-154. doi: 10.1007/s10072-003-0105-3

Baddeley, A. (2000). The episodic buffer: a new component of working memory? Trends Cogn. Sci. 4, 417-423. doi: 10.1016/S1364-6613(00)01538-2

Baddeley, A. D., and Hitch, G. (1974). "Working memory," in The Psychology of Learning and Motivation: Advances in Research and Theory, Vol. 8, ed G. H. Bower (New York, NY: Academic Press), 47-89.

Barabassy, A., Beinhoff, U., and Riepe, M. W. (2007). Cognitive estimation in mild Alzheimer's disease. J. Neural. Transm. 114, 1479-1484. doi: 10.1007/s00702007-0752-2

Barabassy, A., Beinhoff, U., and Riepe, M. W. (2010). Cognitive estimation in aged patients with major depressive disorder. Psychiatry Res. 176, 26-29. doi: 10.1016/j.psychres.2008.06.045

Brand, M., Fujiwara, E., Kalbe, E., Steingass, H. P., Kessler, J., and Markowitsch, H. J. (2003). Cognitive estimation and affective judgments in alcoholic Korsakoff patients. J. Clin. Exp. Neuropsychol. 25, 324-334. doi: 10.1076/jcen.25.3.324.13802 inaccurate answer which was generally interpreted as a clue of pervasive impairment in cognitive estimation (Della Sala et al., 2003). Besides in previous studies relating to cognitive estimation (Appollonio et al., 2003; Khodarahimi and Rasti, 2011) less attention was generally given to bizarre answers (probably due to some difficulties in outlining an operational and actionable definition of the bizarreness index) it could be considered a remarkable clue about the executive domains involvement in the estimation ability: indeed Parkinson's disease (Appollonio et al., 2003), fronto-temporal dementia (Mendez et al., 1998), and focal frontal lesions (Shallice and Evans, 1978; Taylor and O'Carroll, 1995; MacPherson et al., 2014), but also schizophrenia (Khodarahimi and Rasti, 2011) and major depressive disorder patients (Barabassy et al., 2010) reported a poor performance in the cognitive estimation ability, and specifically an higher number of bizarre answers, since the lack of efficacy of executive functions involved in the cognitive estimation process.

\section{Conclusion}

According to the present model, cognitive estimation is neither a pure ability nor a specific measure of executive functions, but it also require e certain amount of semantic knowledge and the ability to access to it. Given this, the Cognitive Estimation Test (Shallice and Evans, 1978 and following versions) may be considered as a useful instrument for the assessment of crystallized intelligence and of cognitive reserve to some extent. We hope this theoretical hypothesis will provide new insights prompting future research toward the understanding of the cognitive estimation process, together with its potential application as an instrument for assessing cognitive reserve.

\section{Acknowledgments}

The present study was approved and funded by IRCCS Istituto Auxologico Italiano.

Cattell, R. B. (1967). The theory of fluid and crystallized general intelligence checked at the 5-6 year-old level. Br. J. Educ. Psychol. 37, 209-224. doi: 10.1111/j.2044-8279.1967.tb01930.x

Cattell, R. B. (1971). Abilities: Their Structure, Growth, and Action. Oxford: Houghton Mifflin.

D’Aniello, G. E., Scarpina, F., Albani, G., Castelnuovo, G., and Mauro, A. (2015). Disentangling the relationship between cognitive estimation abilities and executive functions: a study on patients with Parkinson's disease. Neurol. Sci. doi: 10.1007/s10072-015-2158-5. [Epub ahead of print].

Della Sala, S., MacPherson, S. E., Phillips, L. H., Sacco, L., and Spinnler, H. (2003). How many camels are there in Italy? Cognitive estimates standardised on the Italian population. Neurol. Sci. 24, 10-15. doi: 10.1007/s1007203 00015

Della Sala, S., MacPherson, S. E., Phillips, L. H., Sacco, L., and Spinnler, H. (2004), The role of semantic knowledge on the cognitive estimation task-evidence from Alzheimer's disease and healthy adult aging. J. Neurol. 251, 156-164. doi: 10.1007/s00415-004-0292-8

Franchow, E. I., Suchy, Y., Thorgusen, S. R., and Williams, P. (2013). More than education: openness to experience contributes to cognitive reserve in older adulthood. Aging Sci. 1:109. doi: 10.4172/2329-8847. 1000109 
Goldstein, K. H., and Scheerer, M. (1941). Abstract and concrete behavior: an experimental study with special tests. Psychol. Monogr. 53, 239. doi: 10.1037/h0093487

Hobson, P., Meara, J., and Taylor, C. (2007). The Weigl Colour-Form Sorting Test: a quick and easily administered bedside screen for dementia and executive dysfunction. Int. J. Geriatr. Psychiatry 22, 909-915. doi: 10.1002/gps.1765

Horacek, J., Preiss, M., Tintera, J., Laing, H., Kopecek, M., Spaniel, F., et al. (2010). A functional magnetic resonance imaging study of the cognitive estimation. Act. Nerv. Super Rediviva. 52, 187-192.

Khodarahimi, S., and Rasti, A. (2011). Cognitive estimation in patients with Alzheimer's disease and schizophrenia. J. Neurosci. Behav. Health, 3, 27-31.

Levinoff, E. J., Phillips, N. A., Verret, L., Babins, L., Kelner, N., Akerib, V., et al. (2006). Cognitive estimation impairment in Alzheimer disease and mild cognitive impairment. Neuropsychology 20, 123-132. doi: 10.1037/08944105.20.1.123

MacPherson, S. E., Wagner, G. P., Murphy, P., Bozzali, M., Cipolotti, L., and Shallice, T. (2014). Bringing the cognitive estimation task into the $21 \mathrm{st}$ century: normative data on two new parallel forms. PLoS ONE 9:92554. doi: 10.1371/journal.pone.0092554

Mendez, M. F., Doss, C. D., and Cherrier, M. M. (1998). Use of the Cognitive Estimation Test to discriminate frontotemporal dementia from Alzheimer's disease. J. Geriatr. Psychiatry Neurol. 11, 2-6. doi: 10.1177/089198879801100102

Roldán-Tapia, L., García, J., Cánovas, R., and León, I. (2012). Cognitive reserve, age, and their relation to attentional and executive functions. Appl. Neuropsychol. Adult 19, 2-8. doi: 10.1080/09084282.2011.595458

Sachdev, P. S., and Valenzuela, M. (2009). Brain and cognitive reserve. Am. J. Geriatr. Psychiatry 17, 175-178. doi: 10.1097/JGP.0b013e318196a661

Shallice, T., and Evans, M. E. (1978). The involvement of the frontal lobes in cognitive estimation. Cortex 14, 294-303. doi: 10.1016/S0010-9452(78)80055-0

Spreen, O., and Strauss, E. (1998). A Compendium of Neuropsychological Tests: Administration, Norms, and Commentary. New York, NY: Oxford University Press.
Stern, Y., What is cognitive reserve? (2002). Theory and research application of the reserve concept. J. Int. Neuropsychol. Soc. 8, 448-460. doi: $10.1017 / \mathrm{S} 1355617702813248$

Stern, Y. (2007). Cognitive Reserve: Theory and Applications. Philadelphia, PA: Taylor \& Francis.

Stern, Y. (2009). Cognitive reserve. Neuropsychologia 47, 2015-2028. doi: 10.1016/j.neuropsychologia.2009.03.004

Stern, Y., Alexander, G. E., Prohovnik, I., and Mayeux, R. (1992). Inverse relationship between education and parietotemporal perfusion deficit in Alzheimer's disease. Ann. Neurol. 32, 371-375. doi: 10.1002/ana.4103 20311

Sumowski, J. F., Wylie, G. R., DeLuca, J., and Chiaravalloti, N. (2010). Intellectual enrichment is linked to cerebral efficiency in multiple sclerosis: functional magnetic resonance imaging evidence for cognitive reserve. Brain 133, 362-374. doi: 10.1093/brain/awp307

Taylor, R., and O'Carroll, R. (1995). Cognitive estimation in neurological disorders. Br. J. Clin. Psychol. 34, 223-228.

Tucker, A. M., and Stern, Y. (2011). Cognitive reserve in aging. Curr. Alzheimer Res. 8, 354-360. doi: 10.2174/156720511795745320

Wechsler, D. (1997). Wechsler Adult Intelligence Scale, 3rd Edn. San Antonio, TX: The Psychological Corporation.

Conflict of Interest Statement: The authors declare that the research was conducted in the absence of any commercial or financial relationships that could be construed as a potential conflict of interest.

Copyright (c) 2015 D'Aniello, Castelnuovo and Scarpina. This is an open-access article distributed under the terms of the Creative Commons Attribution License (CC BY). The use, distribution or reproduction in other forums is permitted, provided the original author(s) or licensor are credited and that the original publication in this journal is cited, in accordance with accepted academic practice. No use, distribution or reproduction is permitted which does not comply with these terms. 